\title{
Structural Modeling, Evolution and Ligand Interaction of KMP11 Protein of Different Leishmamiastrains
}

\author{
Ganesh Chandra Sahoo ${ }^{1 *}$, Mukta Rani ${ }^{1}$, Manas Ranjan Dikhit ${ }^{1}$, \\ Waquar Akhtar Ansari ${ }^{1}$, Pradeep Das ${ }^{1}$ \\ ${ }^{1}$ BioMedical Informatics Division, Rajendra Memorial \\ Research Institute of Medical Sciences, Agam Kuan, Patna-800007, India \\ *Corresponding author: Dr. Ganesh Chandra Sahoo, Scientist, BioMedical Informatics Division, \\ Rajendra Memorial Research Institute of Medical Sciences(RMRIMS) Agam Kuan, Patna-800 007 \\ India, Tel: +919973935036; E-mail: ganeshiitkgp@gmail.com, ganeshcs@icmr.org.in \\ Received February 10, 2009; Accepted April 20, 2009; Published April 22, 2009
}

Citation: Sahoo GC, Rani M, Dikhit MR, Ansari WA, Das P (2009) Structural Modeling, Evolution and Ligand Interaction of KMP11 Protein of Different Leishmania Strains. J Comput Sci Syst Biol 2: 147-158. doi:10.4172/jcsb.1000026

Copyright: (C) 2009 Sahoo GC, et al. This is an open-access article distributed under the terms of the Creative Commons Attribution License, which permits unrestricted use, distribution, and reproduction in any medium, provided the original author and source are credited.

\section{Abstract}

The kinetoplastid-specific KMP11 protein was first described for Leishmania donovani associated with the lypophosphoglycan molecule and is localized mainly around the flagellum and flagellar pocket. This protein is well conserved among kinetoplastids and plays an analogous role in all the flagellates, irrespective of their pathogenicity in humans. The structural elucidation of this important protein may bring about information required to target KMP11 to find valid drug candidates. The atomic-resolution model of KMP11 protein of six different Leishmania strains has been determined from its amino acid sequence by using homology modeling. The stereochemical validation of modeled protein has been done by PROCHECK and Profiles-3D scores. The ligand protein interaction of the KMP11 protein models were carried out with several anti-leishmanial drugs i.e. miltefosine, sitamaquine, pentamidine, amphotericin B, SAG (sodium antimony gluconate), leishmanial peptide, paromomycin and vinblastine and an anticancer compound, sulforaphane. Glutamic acid (E) and lysine (K) of KMP11 are the key amino acids during ligand-receptor interaction. From structural and docking analyses, it is hypothesized that KMP11 of a specific Leishmania strain interacts with a specific anti-leishmanial drug candidate i.e. miltefosine interacts only with KMP11 of $L$. braziliensis but not with KMP11 of any other Leishmania strain. Highest docking score was found in case of pentamidine. Anticarcinogenic compound, sulphoraphane has shown comparable docking scores and H-bonds with KMP11 protein of six Leishmania strains.

Keywords: KMP; KMP11; Kinetoplastid membrane protein-11; Anti-leishmanial drug; Pentamidine; Leishmania; Sulforaphane; VL; Kal-Azar; Leishmaniasis; Paromomycin

\section{Introduction}

Tropical disease like Visceral Leishmaniasis (Kala-azar) caused by Leishmania species has become a significant cause of morbidity and mortality in 88 countries (Ashford et al., 1992; Rosypal et al., 2003). It has been reported that VL in adult patients are co-infected with HIV from 33 countries (Rosenthal et al., 2000). Resistance to pentavalent antimonials $[\mathrm{Sb}(\mathrm{V})]$ has been reported earlier even from India which has been the first line of drug of choice for treatment of leishmaniasis (Faraut-Gambbarelli et al., 1997; Lira et al., 1999; Sunder et al., 2000). Second line drugs e.g. pentamidine and amphotericin B has severe side effects and high cost which limit their use (Mishra et al., 1992). Miltefosine (hexadecylphosphocholine) has been approved as the first oral drug for leishmaniasis. It can be used for both antimony-sensitive and antimony-resistant patients (Sunder et al., 1999). In vitro studies have indicated that a single point mutation may lead to miltefosine resistant in the parasite and miltefosine is contra-indicated in pregnancy 


\section{Journal of Computer Science \& Systems Biology - Open Access}

Research Article JCSB/Vol.2 March-April 2009

(Perez-Victoria et al., 2003; Nwaka and Hudson, 2006). Few isoquinolinealkaloids (gyrocarpine, daphnandrine and obaberine) from plant sources have been reported to be effective against Leishmania (Fournet et al., 1988).

The kinetoplastid membrane protein-11 (KMP11) was first described in Leishmania donovani associated to the lipophosphoglycan (LPG) molecule and is located throughout the parasite surface. The $11 \mathrm{kDa}$ molecule was first isolated from Leishmania donovani and its primary structure was determined by protein and DNA sequencing (Jardim et al., 1995). KMP11 protein has a defined cellular localization mainly around the flagellum and flagellar pocket. This protein is well conserved among kinetoplastids and presents numerous universal characteristics such as amino acid composition, cellular localization, and a high expression level in the insect stages. It plays an analogous role in all these flagellates, irrespective of their pathogenicity in humans.

Serological tests have shown that KMP11 may be used to discriminate $L$. chagasi infection from active VL and may serve as a marker of response to therapy (Passos et al., 2005). KMP11 is a potent stimulator of human T cells (Tolson et al., 1994). The complete $L$. donovani KMP11 protein and peptide fractions of the protein have been shown to act as B and $\mathrm{T}$ cell immunogens during visceral leishmaniasis (Jensen et al., 1998).

Evaluation of immune modulatory properties of recombinant antigens kinetoplastid membrane protein-11 (KMP11) and Leishmania homologue of receptors for activated $\mathrm{C}$ kinase (LACK) in cutaneous leishmaniasis (CL) and mucosal leishmaniasis (ML) patients have shown that the modulatory properties of LACK and KMP11 are due to induction of IL-10 production and may be helpful for attenuating chronic inflammatory diseases. However, in some clinical conditions, as demonstrated for ML, these molecules are not able to suppress the IFN-gamma response, even inducing IL-10 production (Carvalho et al., 2005). Another experiment on KMP11 have also shown that upon stimulation with KMP11, mononuclear cells from leishmaniasis patients produces high levels of IL-10, while a predominant IFN-gamma production could be observed in cultures stimulated with $\mathrm{H} 2 \mathrm{~A}$ and soluble Leishmania antigen. KMP11 is recognized by cells and sera of patients with different clinical forms of leishmaniasis, and KMP11, through IL-10 production, proved to be a potent antigen in modulating type 1 immune response (de Carvalho et al., 2003).

Severe neurodegenerative disorders are likely to occur if Leishmania invades into the visceral organs of human. These disorders may not suitably be treated with anti-leishmanial drugs. It is still unknown, how the anti-leishmanial drugs are acting and whether there is any interaction between any of the surface molecules of the parasite and anti-leishmanial drugs. Hence there is requirement of study on structural and functional characteristics of different proteins of Leishmania strains to target the proteins to find novel antileishmanial drug. This research work was carried out with a view to know whether there is any interaction between the anti-leishmanial drugs and the flagellar protein i.e. KMP11 of different Leishmania strains. But x-ray crystallographic structure is not available for this important protein of Leishmania species. The main features of this research work was to generate the three-dimensional (3D) structure of the protein based on the available template structural homologues from protein databank and SCOP database, to validate the models by standard parameters, to know the evolution of this protein in different Leishmania strains and to identify the key amino acids involved in ligand-protein interactions.

\section{Methods}

In this study various three dimensional structural models of the KMP11 protein of different Leishmania strains were generated. The models were validated by Ramachandran plots of PROCHECK and profiles-3D scores of discovery studio software v 2.0. The models of KMP11 were further tested for in silico docking study to know the presence of any interaction between the ligand and KMP11 protein. Various methods applied in this study are given below.

\section{Homology Modeling}

The homology modeling of KMP11 protein of different strains of Leishmania was performed using DS Modeling 2.0. The homologue search and sequence alignment were done by two modules, sequence analysis and protein families. Sequences of eight different strains of Leishmania were identified by searching over NCBI (The National Center for Biotechnology Information) website. Protein families calculate multiple sequence alignment using sequence and structure information, aligns sequences of six (because sequences of $L$. major, L. infantum, L. tropica are identical) different strains of Leishmania and its templates. The final 3-D model was generated by MODELER program of Discovery Studio2.0 which includes automated homology modeling and loop modeling.

\section{Protein Simulation}

KMP11 protein of six different strains of Leishmania models could be further refined by CHARMm (Brooks et 
al., 1983) in DS Modeling 2.0, which provides powerful mechanics and dynamics protocols for studying the energetics and motion of molecules, from small ligands to multicomponent physiological complexes. CHARMm force field (Accelrys) was used throughout the simulation. Constraint was applied to allow only binding site and ligand to be flexible during the simulation.

\section{Protein-ligand Interaction Study:}

This study was done by LigandFit / LigandScore (Venkatachalam et al., 2003) in DS Modeling 2.0, which is an automated tool for protein - small molecule docking/ scoring, including:

- Define binding site (ligand-based or cavity-based).

- Generate ligand conformations (Monte Carlo trials).

- Dock each conformation (align shapes of ligand to binding site; 24 orientation of ligand; Rigid Body Energy minimization (RBM) with grid-based energy function).

- Save the top docked structures (diverse poses).

- Apply scoring function(s) to each docked structure for the best binding mode (binding affinity prediction).

\section{Mathematical Formula for Ligandfit Score (DS)}

Dock Score $($ force field $)=-$ (ligand/receptor interaction energy + ligand internal energy).

There are two energy terms in the forcefield version of DockScore, internal energy of the ligand and the interaction energy of the ligand with the receptor. The interaction energy is taken as the sum of the van der Waals energy and electrostatic energy. The computation of the interaction energy can be quite time consuming. To reduce the time needed for this calculation, a grid-based estimation of the ligand/receptor interaction energy is employed. The van der Waals component of the force field interaction energy typically exhibits a steep rise at short interatomic distances, which can have undesirable consequences in the context of ligand-receptor docking. In particular, the combination of approximating the receptor structure as rigid and limited sampling of ligand conformational space tends to overly penalize poses with "mild" short contacts between the ligand and receptor, due to the "hard" nature of the van der Waals potential as defined in most standard force fields. To overcome this tendency, a softened form of the van der Waals potential is employed with the DockScore function. This softened potential rises to a large but finite value at zero interatomic separation. To maintain a proper balance between electrostatics and van der Waals, the electrostatic energy is also softened to prevent it from dominating the van der Waals energy at short separations. The internal energy of the ligand is computed when using the force field version of Dock Score. The purpose of including the internal energy is to avoid ligand conformations with bad internal nonbond clashes. By default, only the standard (not softened) van der Waals energy is used for the ligand internal energy. Including electrostatic energy as part of the ligand internal energy is optionally available.

Different structural models of KMP11 protein of various Leishmania strains were docked with different anti-leishmanial drugs; both presently used drugs and previously used drugs for the treatment of leishmaniasis. All the ligand structures were downloaded from PubChem database as *.sdf file.

Docking experiments were also performed in GOLD software using the default GOLD fitness function (VDW $=4.0$, $\mathrm{H}$-bonding $=2.5$ ) and evolutionary parameters: population size $=100 ;$ selection pressure $=1.1 ;$ \# operations $=100,000$; $\#$ islands $=5 ;$ niche size $=2 ;$ migration $=10 ;$ mutation $=95$; crossover $=95$ (Jones et al., 1997). Scoring function "Goldscore" was used for evaluation of different docking.

\section{Mathematical Formula for GOLD Docking}

Fitness $=\mathrm{S}\left(\mathrm{hb} \_\mathrm{ext}\right)+1.3750 * \mathrm{~S}\left(\mathrm{vdw} \_\mathrm{ext}\right)+\mathrm{S}\left(\mathrm{hb} \_\right.$int $)$ $+1.0000 * \mathrm{~S}$ (vdw_int).

Five docking runs were performed per structure. If at any time 3 of the 10 poses were within $1.5 \mathrm{~A}^{\circ} \mathrm{RMSD}$ of each other, the docking run for that structure was terminated and docking calculations began for the next structure. Best three poses and docking scores were outputted into a *.mol file and text file respectively.

\section{Result and Discussion}

\section{Structure Prediction and Evolution}

From BLAST and clustal analysis, KMP11 proteins of different Leishmania strains are have ninety percent identity to each other (figure1a). The phylogenetic analysis showed that KMP11 of Leishmania make a separate cluster distinct from Trypanosoma (figure1b). Evolution of KMP11 protein of different Leishmania strains occurred during the same time period.

KMP11 protein has been found to be an essential surface protein of Leishmania. Distant homologues were selected for modeling KMP11 protein using MODELER program. Dali program was run to identify template (Holm et al., 
Journal of Computer Science \& Systems Biology - Open Access

Research Article JCSB/Vol.2 March-April 2009

\begin{tabular}{|c|c|}
\hline Ldonovani & MÅTTYEE FSAKLDRLDQE FNRKMQEQNAKFFADKPD \\
\hline Ltropica & MÄTTYEE FSAKLLRLDEE FNRKMQEQNÄKFFADKPD \\
\hline Lmajor & MẢTTYEE FSAKLDRLDEE FNRKMQEQNAKFFADKF \\
\hline Linfantum & MATTYEE FSAKLDRLDEE FNRKMQEQNAKFFADKH \\
\hline Lamazonensis & MATTYEE FSAKLDRLDEE FNRKMQEQNAKFFADKH \\
\hline Lpanamensis & MÄTTYE FAAKLDRLDEE FNKKMQEQNAKFFAD \\
\hline Lguyanensis & 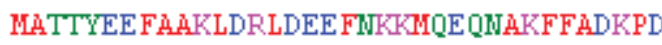 \\
\hline Lbraziliensis & 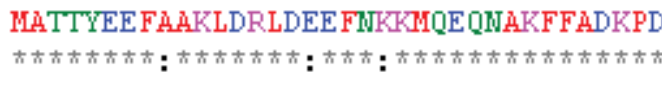 \\
\hline Ldonovani & KFNKKMHEHSEHFKQKFÄELLEQQKÄДОYPSK \\
\hline Ltropica & KFWKKMHEHSEHFKQKFAELLEQQKÄД̊YPSK 92 \\
\hline Lmajor & KFWKKMHEHSEHFKQKFÄLLEQQKAूД̊QYPSK 92 \\
\hline Linfantum & KFWKKMHEHSEHFKQKFÄELLEQQKÄД̆QYPSK 9 \\
\hline Lamazonensis & KFWKKMHEHSEHFKQKFAELLEQQKÄДЗYPSK 92 \\
\hline Lpanamensis & KFNKKMHEHSEHFKHKFAELLEQQKÄДQYPSK 92 \\
\hline Lguyanensis & 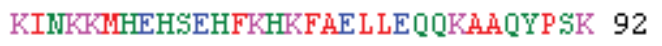 \\
\hline Lbraziliensis & 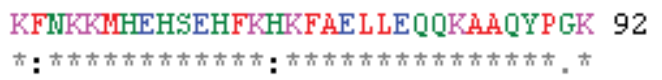 \\
\hline
\end{tabular}

Figure1a: Clustal W shows very close identity among the KMP11 sequence of Leishmania and trypanosome sp.

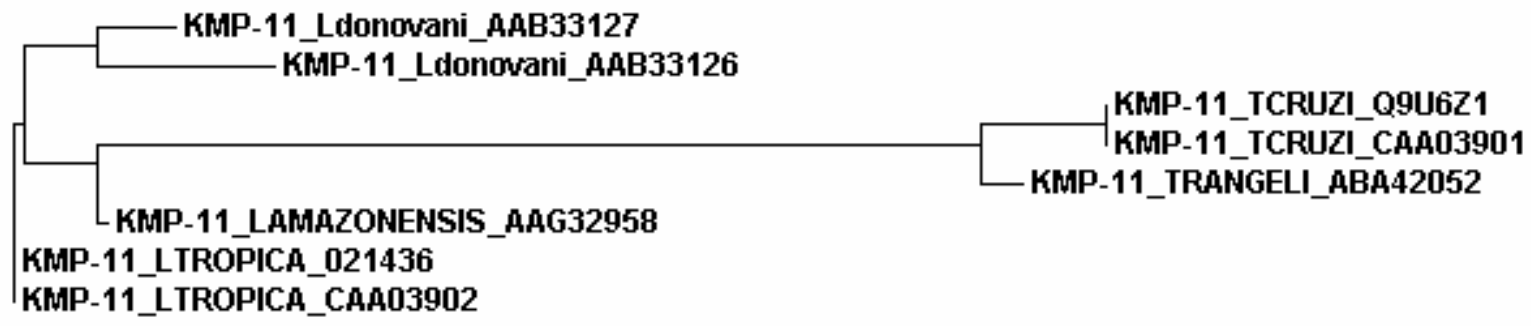

Figure 1b: Phylogram shown here depicts the close analogy among different Leishmania sp \& T. cruzi and T. rangeli

2008). The PDB ids of selected templates were 2odm, 2c5k and 1owa. PDB '2odm' is the crystal structure of S. aureus YlaN, an essential leucine rich protein involved in the control of cell shape. PDB ' $2 \mathrm{c} 5 \mathrm{k}$ ' is crystal structure of N-terminal domain of tlg1 complexed with $\mathrm{N}$-terminus of Vps51. PDB '1owa' is the solution structural studies on human erythrocyte alpha spectrin $\mathrm{N}$ terminal tetramerization domain. PDB templates '1nkp' and '1am9' were also detected to be distant homology of KMP11 from Dompred program (Marsden et al., 2002). Only one domain was detected in KMP11 protein of six different Leishmania strains from Dompred. PDB '1npk' is the X-ray structure of the basic/ helix-loop-helix/leucine zipper (bHLHZ) domains of MycMax and Mad-Max heterodimers bound to their common DNA target (Nair and Burley, 2003). The other PDB ' $1 \mathrm{am} 9$ ' is the three dimensional coordinates of the human SREBP$1 \mathrm{~A}$ bound to LDL receptor promoter. The sterol regulatory element binding proteins (SREBPs) are helix-loop-helix transcriptional activators that control expression of genes encoding proteins essential for cholesterol biosynthesis/ uptake and fatty acid biosynthesis (Párraga et al., 1998).

Helix-turn-helix structure of KMP11 protein of different Leishmania strains is revealed from the modeling experi- ments (figure $2 \mathrm{a}$ and figure $2 \mathrm{~b}$ ). Amino acids 2-35 and 4286 are responsible for formation of first helix and second helix respectively. Validation of different models of KMP11 protein was carried out with Ramachandran plot computed by PROCHECK program which showed $96.4 \%$ residues in favored and allowed regions (figure $2 \mathrm{c}$ ). No invalid region was found in the modeled structures of KMP11 protein. Side chain refinement and loop modeling did not yield any further change in the models.

\section{Ligand Protein Interaction}

Ligand-protein interaction between KMP11 and antileishmanial drugs hasn't yet been reported in any research work. For this purpose, docking study has been carried out by LigandFit tool of DS (Accelrys) and GOLD. Different ligand binding sites in the KMP11 protein were detected. Different binding conformations of the ligands (antileishmanial drugs) with the protein, scored by LigandFit score were also detected during the study. More than two fifty dockings has been performed in DS for finding ligand protein interactions of various ligands taken for this study. Different ligands selected for this study includes miltefosine, sitamaquine, pentamidine, amphotericin B, SAG (sodium 
Journal of Computer Science \& Systems Biology - Open Access

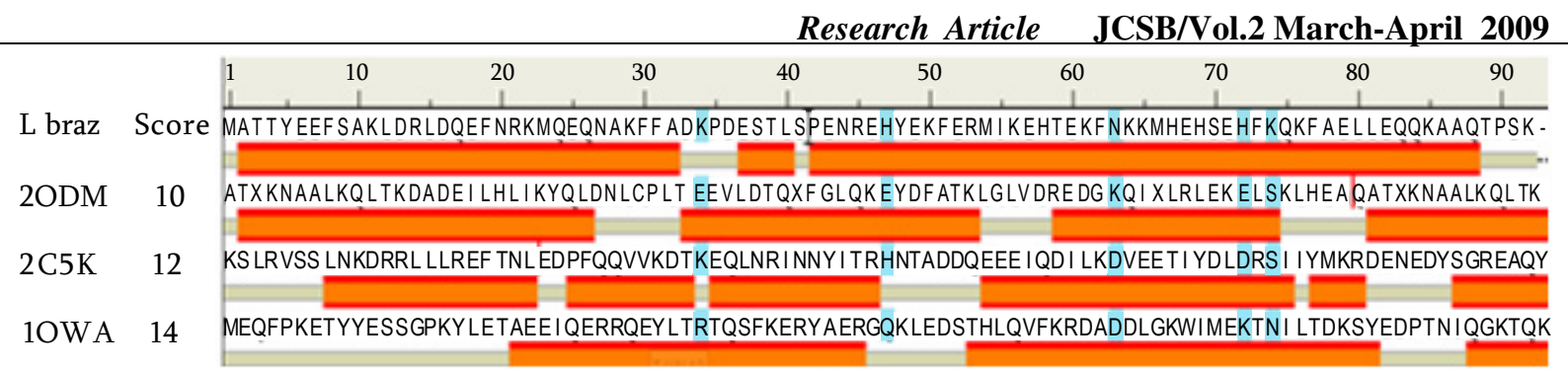

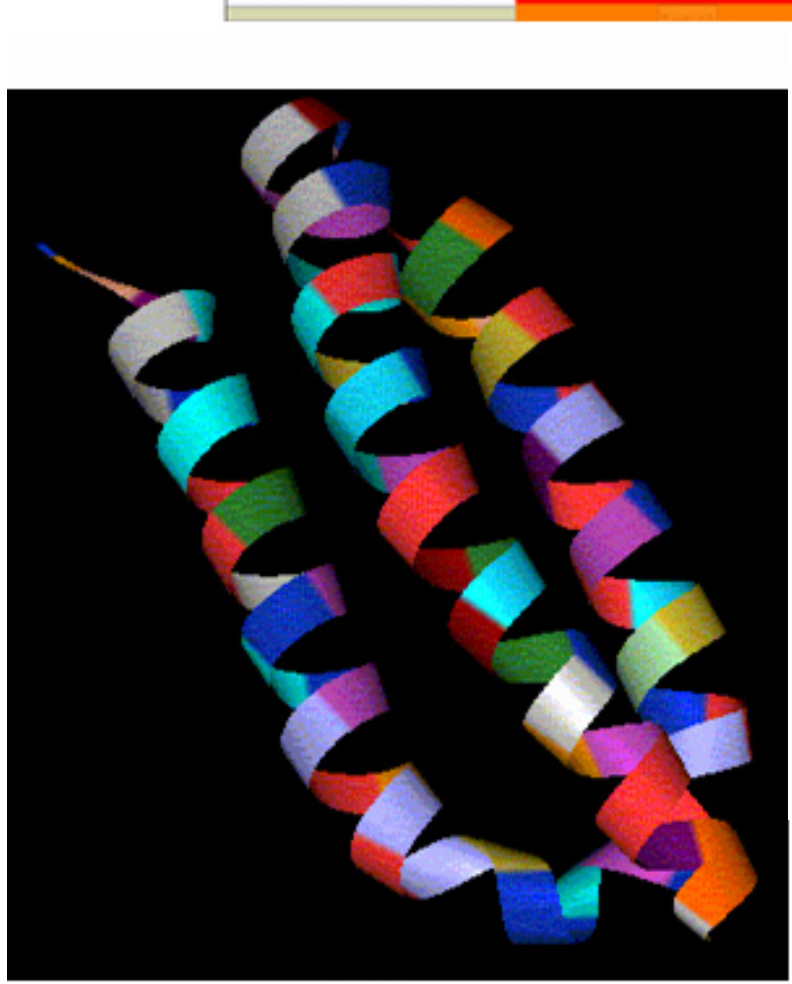

2b 2a

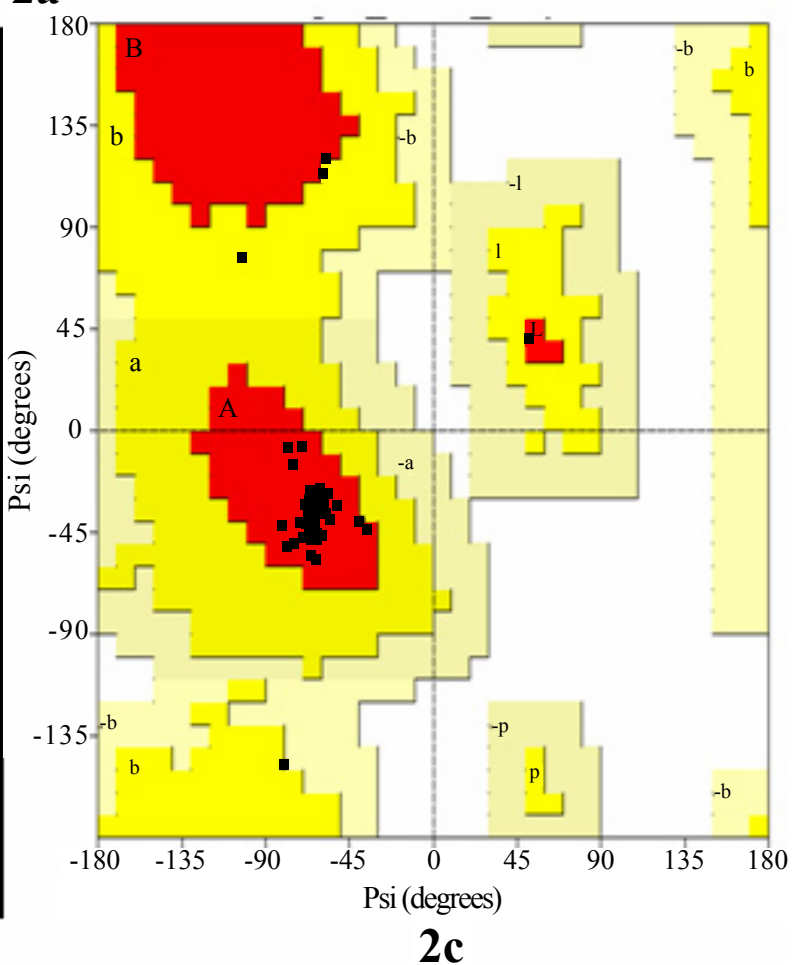

Figure 2a: Alignment of templates and target (KMP11 protein of L. braziliensis) performed by Discovery Studio 2.0. Predicted secondary structure of KMP-11 protein of Leishmania strains of known three dimensional structures. Helices are colored red, superimpose in blue.

Figure 2b: A screenshot (from DS) of predicted alpha helical structure of KMP11 protein of L. braziliensis.

Figure 2c: Ramachandran plot for the model of KMP11 protein of L. major (96.4\% amino acid of the model are in favored and allowed region).

antimony gluconate), leishmanial peptide, paromomycin, vinblastine, sulforaphane, ketoconazole and allopurinol.

It is interesting to note that the antileishmanial drug, pentamidine was able to find five different binding sites in KMP11 protein of various Leishmania strains e.g. L. major, L. donovani, L. braziliensis, L. amazonensis, $L$. panamensis and L. guyanensis. In some binding sites of KMP11 protein, ten different binding conformations of pentamidine have been observed.

Vinblastine is reported to be down regulating mRNA level of KMP11 in Trypanosoma cruzi. Vinblastine have no interaction with the KMP11 protein of different Leishmania strains as no ligand binding site has yet been found in this study. Hence it is known from this in silico study that vinblastine don't have any effect on KMP11 protein phase but may have effect at RNA phase (Thomas and Garcia, 2000). Similarly amphotericin B, SAG (sodium antimony gluconate) and leishmanial peptide did not find any ligand binding site in this flagellar protein, KMP11.

In case of L. braziliensis, four antileishmanial drugs e.g. miltefosine, sitamaquine, pentamidine and paromomycin showed high dock scores, in case of both GOLD score and DS dock score, which signifies that these four compounds have high affinity for KMP11. KMP11 protein of L. braziliensis shows highest ligand protein interaction with antileishmanial drug, pentamidine (figure 3 and figure 4). No other ligand has shown any binding affinity for the modeled KMP11 protein. Ten different binding conformations have been observed during docking study in DS (Accelrys) in case of the above mentioned four drugs. Paromomycin is likely to form two H-bonds with lysine $\left(74^{\text {th }}\right)$ 


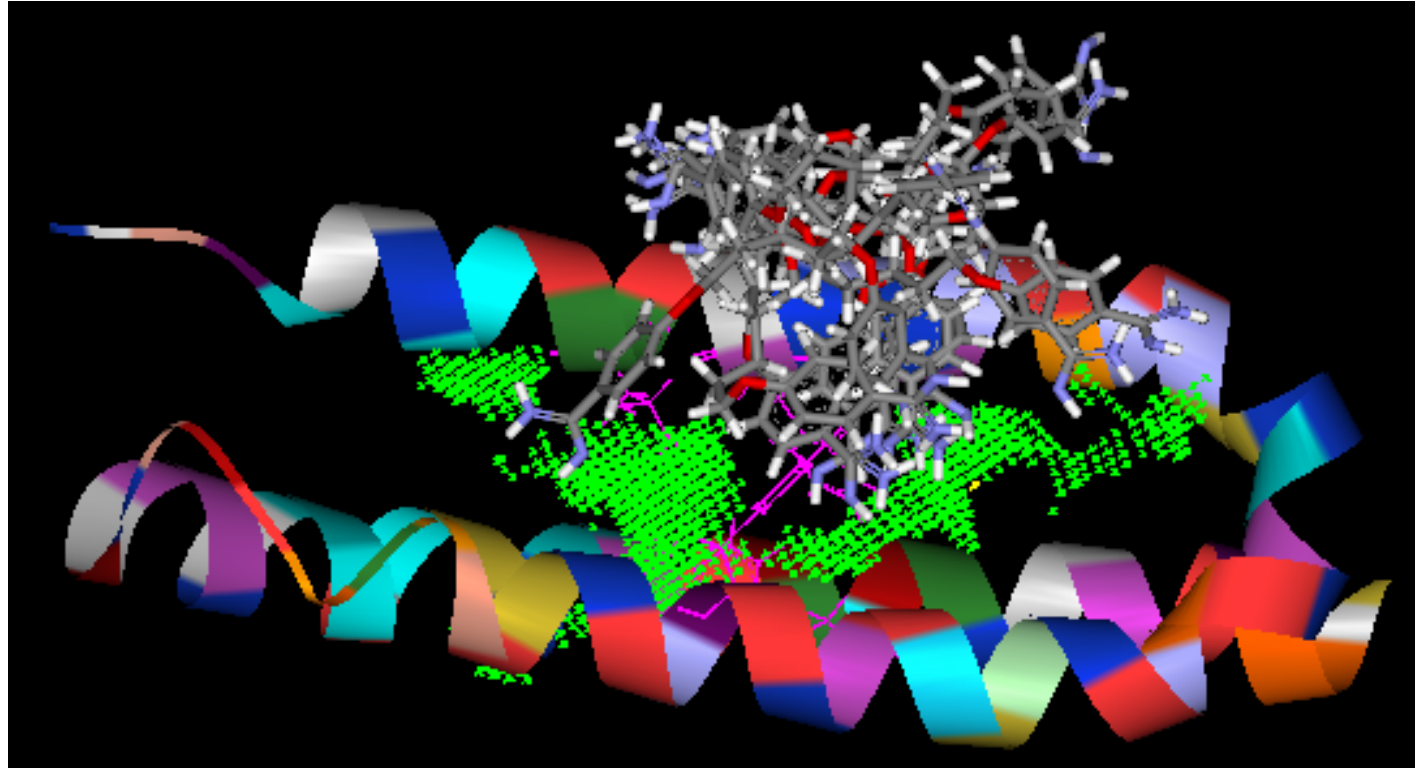

Figure 3: A screenshot from docking of KMP11 protein of L. braziliensis with an antileishmanial drug, pentamidine. Different binding modes of pentamidine are shown. Green mesh part is the ligand binding site found using ligand receptor interaction tool of Discovery Studio tool(Structure-Flat Ribbons, Hide Sphere, Color, decolor Residue Atoms within Sphereactivate, and Hide residue around sphere).

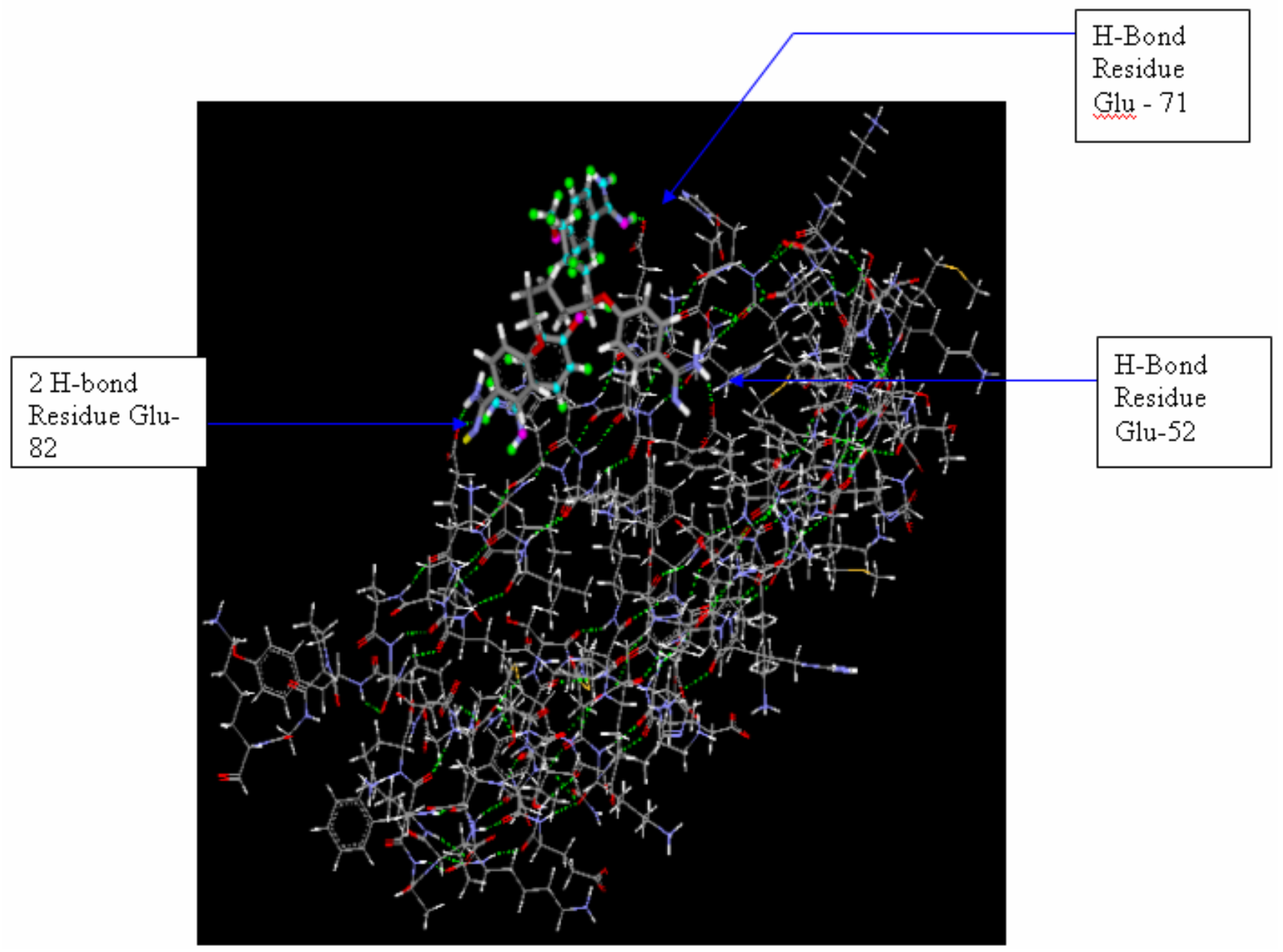

Figure 4: Screenshot from docking of L. braziliensis with pentamidine (having highest dock score 80.939) with ligandreceptor interaction tool of Discovery Studio (Accelrys). It shows three H-bonds formed between different atoms of ligand and amino acids of KMP11 protein of L. braziliensis i.e. involved in Glu-52, Glu-71 and Glu (E)-82. 
and asparagine $\left(63^{\text {rd }}\right)$ amino acids. Three amino acids e.g. Glu-52, Glu-71 and Glu-82 of one helix of the modeled KMP11) were found to be involved in formation of $\mathrm{H}$-bonds with this ligand (figure 4). Hence glutamic acid (E) of this flagellar protein (KMP11) is the key amino acid responsible for ligand protein interaction in L. braziliensis. Two different ligand binding sites in KMP11 protein are known to be present for pentamidine only. It is interesting to be noted that one pose of pentamidine has highest ligandfit score (80.939) whereas second conformation of pentamidine didn't show any affinity for KMP11 protein of $L$. braziliensis as it showed very low ligandfit score (7.06). It's well known that hydrogen bond plays an important role for the structure and function of biological molecules, especially for inhibition in a complex. Hence pentamidine is likely to be the best antileishmanial drug in case of $L$. braziliensis. It has also been reported in other research works that pentamidine is an aromatic diamidine that displays multiple effects and is active in vitro against a number of different bacteria, protozoa, and fungi.

In case of L. donovani, high dock scores have been observed in case of three antileishmanial drugs e.g. sitamaquine, pentamidine and paromomycin. In one of the conformations of ligand paromomycin, four $\mathrm{H}$ - bonds are formed \& amino acid residues involved are H67, E52 (3H- bond, is bifractional). So glutamic acid $\left(52^{\text {nd }}\right)$ and histidine $\left(67^{\text {th }}\right)$ are vital for interaction of paromomycin with KMP11 of $L$. braziliensis. It has been earlier reported that an ointment containing $15 \%$ paromomycin and $0.5 \%$ gentamicin shows $100 \%$ effective in BALB/c mice (Grogl et al., 1999). Paromomycin is a broad-spectrum aminoglycosidic antibiotic. Hence it is predicted that paromomycin may be specifically acting against $L$. donovani as an antileishmanial drug i.e. acting as an antagonist to KMP11 rather than agonist. It has been earlier reported that paromomycin along with gentamycin is $100 \%$ effective in BALBc mice.

KMP11 of L. amazonensis and L. guyanensis showed interaction only with few conformations of pentamidine but it doesn't show any interaction with other compounds or antileishmanial drugs. In case of $L$. panamensis only one ligand binding site was found, which showed docking with four antileishmanial drugs e.g. pentamidine, paromomycin, miltefosine and sitamaquine. These drug candidates have also proved high GOLD scores and ligandfit scores (DS).

From GOLD program, different number of H-bonds involved in docking and docking scores of antileishmanial drugs with KMP11 protein of different Leishmania strains has been shown in figure 5. Pentamidine have shown highest (51.42) GOLD score with KMP11 of L. guyanensis.

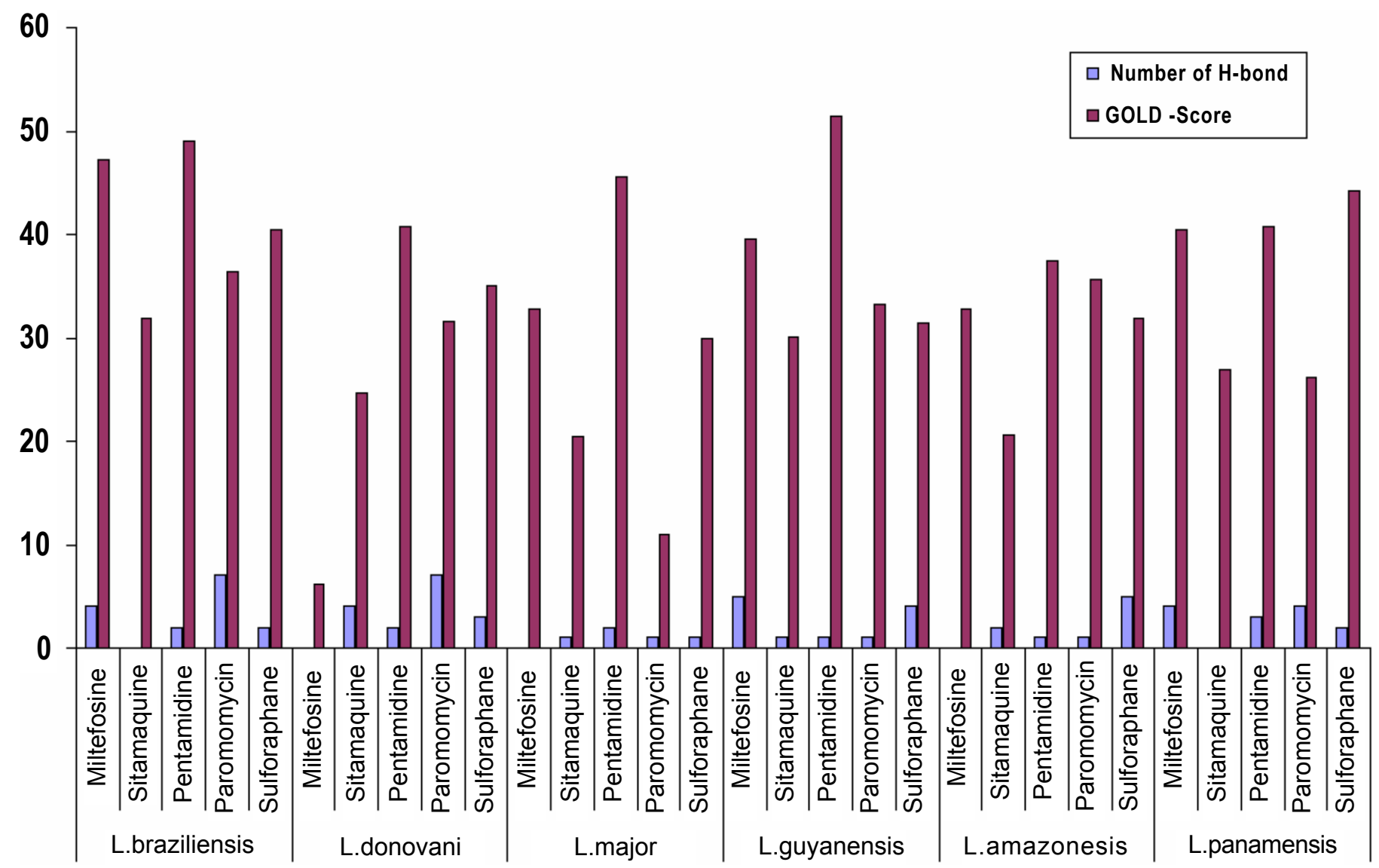

Figure 5: Bar diagram showing GOLD-scores and involved H-bonds between flagellar protein, KMP11 of different Leishmania strains and different ligands (antileishmanial drugs and sulphoraphane) 


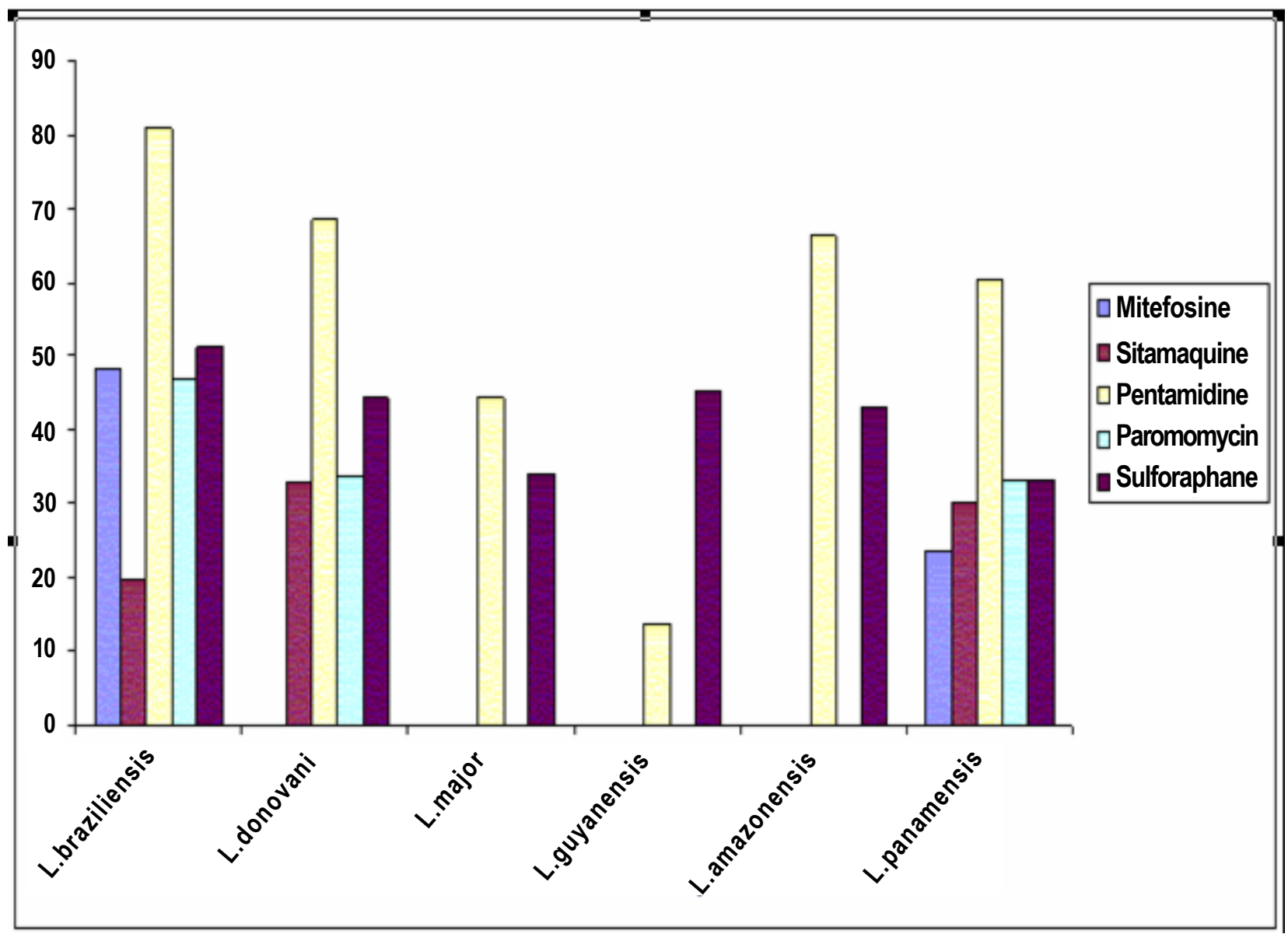

Figure 6: Bar diagram showing comparative Ligandfit score (DS2.0) of different ligands (implicated for the treatment of leishmanaisis) with KMP11 protein of six different Leishmania strains.

Pentamidine have also shown second highest score (49.04) with KMP11 of L. braziliensis. Highest number (7) of Hbond involved during docking has been observed in case of paromomycin with KMP11 of L. braziliensis and $L$. donovani. In few cases there is no involvement of H-bonds e.g. sitamaquine with KMP11 of L. braziliensis and $L$. panamensis, miltefosine with KMP11 of $L$. donovani and L. amazonensis. Various amino acids at different positions of KMP11 protein have been found to be essential for ligand protein interaction with implicated antileishmanial drugs. Among them four amino acids namely glutamic acid (E), threonine $(\mathrm{T})$, lysine $(\mathrm{K})$ and methionine $(\mathrm{M})$ have been identified to be key participants of KMP11 protein of different Leishmania strains.

Sulforaphane, an anticancer compound, first isolated from broccoli (Zhang et al., 1992) was taken for ligand protein interaction study to know whether it has any comparable number of H-bonds and docking scores to the implicated drugs used for the treatment of leishmaniasis. This compound has shown comparable results, which is learned from the bar diagram considering the number of involved $\mathrm{H}$ bonds in docking of KMP11 protein of each Leishmania strain with different antileishmanial drugs and docking scores (GOLD) depicted in figure 5. Highest GOLD score (44.24) has been observed in case of L. panamensis, which is more than or comparable to the docking scores of currently implicated drugs for the management of leishmanaisis. Highest number (5) of $\mathrm{H}$-bonds between KMP11 and sulforaphane has been found in case of $L$. amazonensis, which is the second lowest.

Ligand-protein interaction study of sulforaphane in discovery studio have shown highest dock score (51.31) with KMP11 of L. braziliensis (figure 6). The superimposition of ten different conformations of sulforaphane with ligand binding amino acids of KMP11 protein of L. braziliensis is shown in (figure 7). The labeled amino acids also form $\mathrm{H}$ bond with the concerned ligand. From the figure 6 it is known that sulforaphane is the second ligand having affinity to KMP11 of all Leishmania strains considered in this study. Sulforaphane is the second ligand having highest ligandfit score. Four ligand binding sites in KMP11 and ten different conformations of sulforaphane have been detected from discovery studio. In case of $L$. major, sulforaphane binds to KMP11 in three different poses. The interesting thing is that the involved amino acid responsible for formation of H-bond is lysine (LYS29, LYS85 and LYS92) with 


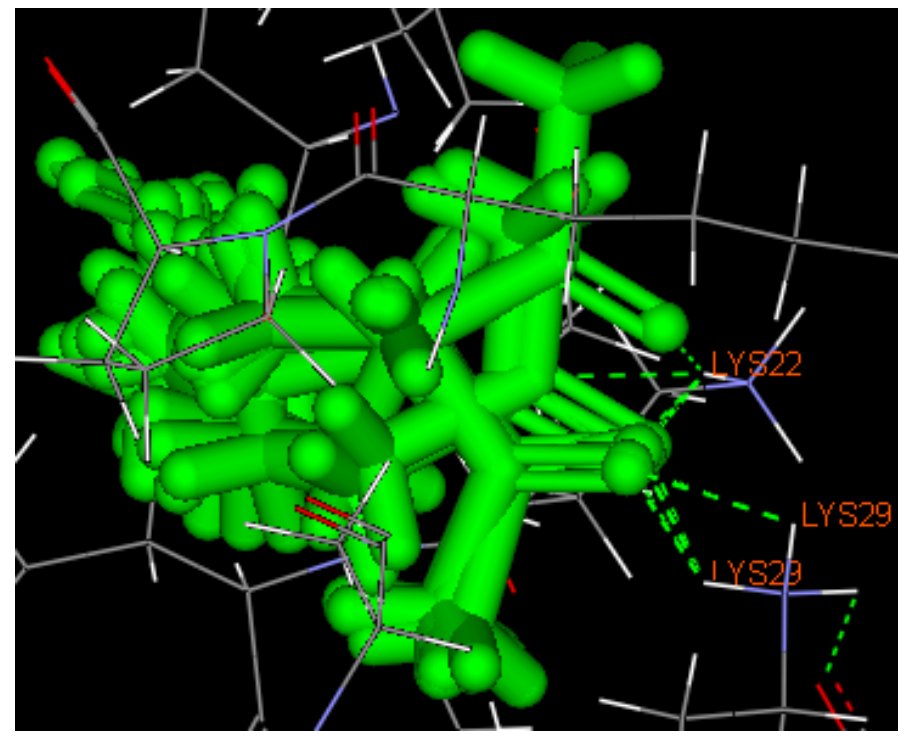

Figure 7: The superimposition of ten different conformations of sulforaphane showing ligand binding amino acids of KMP11 protein of L. braziliensis involved in ligand protein interaction. It was found that the output is in agreement with the surface model generated by Ligandfit.

\begin{tabular}{|c|c|c|c|c|c|c|c|c|c|}
\hline $\begin{array}{l}\text { Leishmania } \\
\text { Strains }\end{array}$ & Ligands & LigScore1 & LigScore2 & PLP1 & PLP2 & Jain & PMF & $\begin{array}{l}\text { Dock } \\
\text { score }\end{array}$ & $\begin{array}{c}\text { GOLD- } \\
\text { Score }\end{array}$ \\
\hline \multirow[t]{5}{*}{ L. braziliensis } & Pentamidine & 2.28 & 1.4 & -4.36 & 0.25 & $-\overline{2.62}$ & 9.18 & 80.939 & 49.04 \\
\hline & Sulforaphane & 1.67 & 2.28 & 47.55 & 47.84 & 3.26 & 25.61 & 51.31 & 40.51 \\
\hline & Miltefosine & 1.31 & 2.49 & 8.47 & 11.06 & $\begin{array}{l} \\
5.44\end{array}$ & 25.16 & 48.28 & 47.27 \\
\hline & Sitamaquine & 0.6 & 3.03 & 31.33 & 30.63 & $\begin{array}{l}- \\
2.03\end{array}$ & 57.15 & 19.8 & 31.84 \\
\hline & Paromomycin & 3.26 & 4.48 & 58.41 & 58.48 & $\begin{array}{l}- \\
1.98\end{array}$ & 92.09 & 46.761 & 36.40 \\
\hline \multirow[t]{4}{*}{ L. donovani } & Pentamidine & 2.34 & 2.15 & 55.26 & 59.24 & 1.2 & 62.71 & 68.616 & 40.70 \\
\hline & Sulforaphane & 1.86 & 2.89 & 25.78 & 27.64 & $\begin{array}{l}- \\
1.04\end{array}$ & 22.85 & 44.314 & 35.02 \\
\hline & Sitamaquine & 1.37 & 4.11 & 50.89 & 48.49 & 0.65 & 66.03 & 32.852 & 24.68 \\
\hline & Paromomycin & 2.29 & 2.05 & 47.02 & 50.93 & $\begin{array}{l}- \\
3.01\end{array}$ & 94.37 & 33.76 & 31.57 \\
\hline \multirow[t]{2}{*}{ L. major } & Pentamidine & 0.44 & 0.97 & 26.82 & 28.01 & 0.47 & 59.99 & 43.44 & 45.49 \\
\hline & Sulforaphane & 1.53 & 2.91 & 15.9 & 19.51 & 1.45 & 25.64 & 36.113 & 29.92 \\
\hline \multirow[t]{2}{*}{ L. guyanensis } & Sulforaphane & 3.18 & 3.11 & 36.89 & 34.08 & 1.36 & 13.38 & 45.476 & 51.42 \\
\hline & Pentamidine & -0.27 & -1.09 & 2.21 & 13.87 & $\begin{array}{l}- \\
0.04\end{array}$ & 31.25 & 13.705 & 42.46 \\
\hline \multirow[t]{2}{*}{ L. amazonensis } & Pentamidine & 1.22 & 2.06 & 37.46 & 43.78 & 1.3 & 68.03 & 66.305 & 37.44 \\
\hline & Sulforaphane & 2.37 & 2.95 & 22.94 & 21.12 & 1.57 & 4.55 & 43.083 & 31.91 \\
\hline \multirow[t]{5}{*}{ L. panamensis } & Pentamidine & 2.24 & 2.08 & 38.52 & 49.05 & 1.17 & 54.69 & 60.289 & 40.71 \\
\hline & Sulforaphane & 1.94 & 2.47 & 22.18 & 22.72 & 0.89 & 19.62 & 32.603 & 44.24 \\
\hline & Miltefosine & 1.4 & 3.01 & 11.78 & 14.6 & $\begin{array}{l}- \\
5.15\end{array}$ & 36.04 & 23.647 & 40.38 \\
\hline & Sitamaquine & 1.55 & 3.48 & 46.43 & 46.82 & 0.54 & 57.56 & 30.084 & 26.99 \\
\hline & Paromomycin & 3.63 & 4.04 & 61.56 & 67.49 & $-\overline{1.86}$ & -0.08 & 32.97 & 26.18 \\
\hline
\end{tabular}

Table 1: Different ligands protein interaction carried out in LigandFit tool of Discovery Studio. Scoring of the docking has been done in different methods i.e. LigScore1, LigScore2, PLP1, PLP2, Jain, PMF and Dock scores. The table represents the highest scores of different ligands interacted with modeled KMP11 protein of six different Leishmania strains.

ISSN:0974-7230 JCSB, an open access journal 


\section{Journal of Computer Science \& Systems Biology - Open Access}

Research Article JCSB/Vol.2 March-April 2009

different poses of sulforaphane. LYS74 in case of $L$. amazonensis, LYS45 in L. panamensis, LYS74 and LYS76 in L. guyanensis are responsible for H-bond formation with sulforaphane. Hence it is hypothesized that sulforaphane, which is available in cruciferous vegetables, such as cauliflower, cabbage and kale, may be implicated for the treatment of leishmaniasis in human beings. This novel compound should be tested in vitro and in vivo for appropriate application in antileishmanial therapy.

A table (table 1) of different docking scores based on different scoring functions has been prepared considering top scoring (highest binding affinity) ligand protein interactions of KMP11 protein of six different Leishmania strains and different compounds including antileishmanial drugs from discovery studio (Accelrys). Different docking scoring functions described in the table are LigScore1 and LigScore2 (Krammer et al., 2005), Piecewise Linear Potential-PLP1 (Gehlhaar et al., 1995), PLP2 (Gehlhaar et al., 1999), Jain (Jain , 1996), PMF (Muegge and Martin, 1999). The se scoring functions tend to fall into two major classes emphasizing either: H-bonding interactions or van der Waals, hydrophobic as well as polar attractive/repulsive interactions. GOLD score, PLP (1\&2), as well as the Monte Carlo scoring functions all have highly weighted H-bonding terms. On the other hand, LigScore (1 \& 2), Jain, PMF, and dock score contain highly weighted terms for van der Waals interactions, lipophilic interactions, and polar attractive/repulsive interactions as well as terms for buried and total polar surface areas. High ligandfit scores have also been observed without $\mathrm{H}$-bonding. As H-bonding appears to play a critical role in binding of different ligands, it is not amazing that the scoring functions with $\mathrm{H}$-bonding terms seemed to do better. It is predicted that the ligands, whose dock scores (DS and GOLD) are more than thirty, are likely to have better ligand-protein interaction with KMP11.

For evaluation of dock scores (GOLD score and dock score in table 1) given by these two tools, we have performed one tailed $t$-test. After the $t$-test, it was found that there is no significant difference between the two scores given by two software tools as the calculated value (0.1391) is less than the tabulated value $\left(t_{.95}=1.7247\right)$ in one tailed two sample unequal variance $t$-test at ninety-five percent confidence interval and degree of freedom $=20$.

\section{Conclusions and Perspectives}

Compounds selected for this study have earlier been reported in other works on leishmaniasis, one compound which hasn't been reported about its antileishmanial activity is sulforaphane. There is no report that these compounds interact with KMP11 protein of Leishmania. This is the first report of ligand protein interaction about the currently used drugs for the treatment of leishmaniasis and KMP11 protein of Leishmania. The study of inhibitory capacity of antileishmanial drugs is a prerequisite for design of novel drug candidates against Leishmania species. Homology modeling of Leishmania KMP11 shed new light on the ligand binding features of this protein. Highest docking score along with more number of $\mathrm{H}$-bonds were taken to be the best inhibitory compound for KMP11 protein of Leishmania.

From ligand protein interaction study, it is learned that few drugs, which have been implicated for leishmaniasis treatment, are specific to specific strain (pentamidine to $L$. braziliensis). Few ligands (e.g. vinblastine) don't have any effect on KMP11. Glutamic acid (E) and lysine (K) of KMP11 are the key amino acids during ligand-receptor interaction. Pentamidine has been found to be interacting with KMP11 protein of different Leishmania strains. Hence it is hypothesized that pentamidine may be the best antileishmanial drug from this KMP11 protein and antileishmanial drug interaction study. Paromomycin have also been found to have best interaction with KMP11 of $L$. donovani (figure 8). Except miltefosine and vincristine,

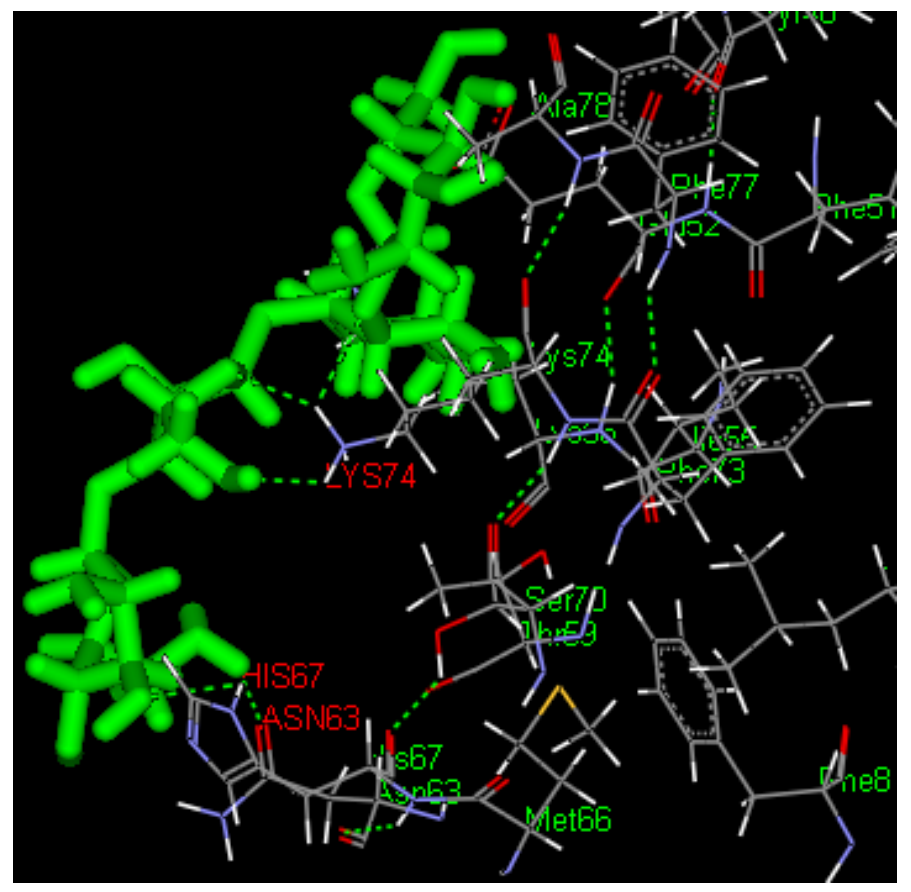

Figure 8: A screenshot from docking of KMP11 protein of $L$. donovani with an antileishmanial drug, paromomycin using ligand receptor interaction tool of Discovery Studio. Large green part represents the structure of paromomycin. Amino acid residues of KMP-11 protein involved in H-bond formation (thin green dotted portion) with paromomycin i.e. His67, Asn63 and Lys74 are labeled in red color. 
another anticancerous drug, sulforaphane available from different cruciferous plants, have shown comparable results and hence may be implicated for the treatment of leishmaniasis. The treatment cost will be very less if sulforaphane proves to be a drug candidate for the treatment of leishmaniasis in comparison to current applied drugs for leishmaniasis as this is available from cruciferous plants and available throughout the world. These in silico study must be proved in laboratory before these antileishmanial drugs or likely to be antileishmanial drugs can be brought into clinical trials. As KMP11 is localized in the flagellum and flagellar pocket of Leishmania (which can be ascertained by fluorescent microscopic studies) the inhibition efficacy of the above mentioned compounds can be studied by incubating the insectal stage of Leishmania culture with various ligands taken in this study and measuring the IC50 values.

\section{Acknowledgments}

This study was supported by a grant for setting up biomedical informatics centre from Indian Council of Medical Research (ICMR), Govt. of India. We acknowledge Dr. Sindhu Prava Rana, Mukesh Kumar, Narendra Tiwari for helping us in preparation of the manuscript. We thank Dr. Meera Singh, Scientist F of ICMR, New Delhi for helping us in setting up our biomedical informatics dept in RMRIMS, Patna, India.

\section{References}

1. Ashford RW, Desjeux P, Deraadt P (1992) Estimation of population at risk of infection and number of cases of Leishmaniasis. Parasitol Today 8: 104-105. "CrossRef » Pubmed » Google Scholar

2. Brooks BR, Bruccoleri RE, Olafson BD, States DJ, Swaminathan S, et al. (1983) CHARMM: A program for macromolecular energy, minimization, and dynamics calculations. J Comp Chem 4: 187-217. "CrossRef » Google Scholar

3. Carvalho LP, Passos S, Dutra WO, Soto M, Alonso C, et al. (2005) A Effect of LACK and KMP11 on IFN-gamma production by peripheral blood mononuclear cells from cutaneous and mucosal leishmaniasis patients. Scand J Immunol 61: 337-342. » CrossRef » Pubmed » Google Scholar

4. de Carvalho LP, Soto M, Jerônimo S, Dondji B, Bacellar O, et al. (2003) Characterization of the immune response to Leishmania infantum recombinant antigens. Microbes Infect 5: 7-12. " CrossRef » Pubmed " Google Scholar

5. Faraut Gambarelli F, Pioroux R, Deniau M, Giusiano B, Marty P, et al. (1997) In vitro resistance of Leishmania infantum
Research Article JCSB/Vol.2 March-April 2009

to meglumine antimoniate: a study of 37 strains collected from patients with visceral leishmaniasis. Antimicrob Agents Chemother 41: 827-830. " CrossRef » Pubmed » Google Scholar

6. Fournet A, Muñoz V, Manjon AM, Angelo A, Hocquemiller R, et al. (1988) Active antihelminitic alkaloids: active in vitro against Leishmanic Tropica the protozoa involved in leishmaniasis. J Ethnopharmacol 24: 327-335. » CrossRef » Pubmed » Google Scholar

7. Gehlhaar DK, Verkhivker GM, Rejto PA, Sherman CJ, Fogel DB, et al. (1995) Molecular Recognition of the Inhibitor AG-1343 by HIV-1 Protease: Conformationally Flexible Docking by Evolutionary Programming. Chem Biol 2: 317-324. » CrossRef » Pubmed » Google Scholar

8. Gehlhaar DK, Bouzida D, Rejto PA, Parrill L, Rami RM (1999) Rational drug design: novel methodology and practical applications. American Chemical Society Washington 292.

9. Grogl M, Schuster BG, Ellis WY, Berman JD (1999) Successful topical treatment of murine cutaneous leishmaniasis with a combination of paromomycin (aminosidine) and gentamicin. J Parasitol 85: 354-359. »CrossRef » Pubmed » Google Scholar

10.Jardim A, Funk V, Caprioli RM, Olafson RW (1995) Isolation and structural characterization of the Leishmania donovani kinetoplastid membrane protein-11, a major immunoreactive membrane glycoprotein. Biochem J 305: 307-313. » CrossRef » Pubmed » Google Scholar

11.Jardim A, Hanson S, Ullman B, McCubbin WD, Kay CM, et al. (1995) Cloning and structure-function analysis of the Leishmania donovani kinetoplastid membrane protein-1 1. Biochem J 305: 315-320. » CrossRef » Pubmed » Google Scholar

12.Jain AN (1996) Scoring noncovalent protein-ligand interactions: A continuous differentiable function tuned to compute binding affinities. J Comput Aided Mol Des 10: 427-440.» CrossRef » Pubmed » Google Scholar

13.Jensen AT, Gasim S, Ismail A, Gaafar A, Kurtzhals JA, et al. (1998) Humoral and cellular immune response to synthetic peptides of the Leishmania donovani kinetoplastid membrane protein-11. Scand J Immunol 48: 103-109.» CrossRef » Pubmed » Google Scholar

14.Jones G, Willett P, Glen RC, Leach AR, Taylor R(1997) Development and validation of a genetic algorithm for flexible docking. J Mol Biol. 267:727-748. "CrossRef " Pubmed " Google Scholar

15. Holm L, Kriinen S, Rosenstrm P, Schenkel A (2008) 


\section{Journal of Computer Science \& Systems Biology - Open Access}

Research Article JCSB/Vol.2 March-April 2009

Searching protein structure databases with DaliLite v.3. Bioinformatics 24: 2780-2781. » CrossRef » Pubmed » Google Scholar

16. Montes M, Miteva MA, Villoutreix BO (2005) Structure-based virtual ligand screening with LigandFit: Pose prediction and enrichment of compaund collections. J Mol Graph Model 23: 395.

17.Lira R, Sunder S, Makharia A, Kenney R, Gam A, et al. (1999) Evidence that high incidence of treatment failure in Indian kala-azar is due to the emergence of antimony resistant strains of Leishmania donovani. J Infect Dis 180: 564-567. » CrossRef » Pubmed » Google Scholar

18. Mishra M, Biswas UK, Jha DN, Khan AB (1992) Amphotericin versus pentamidine in antimony-unresponsive kalaazar. Lancet 340: 1256-1257. " CrossRef » Pubmed " Google Scholar

19. Muegge I, Martin YC, (1999) A General and Fast Scoring Function for Protein-Ligand Interactions: A Simplified Potential Approach. J Med Chem 42: 791-804.

" CrossRef » Pubmed » Google Scholar

20.Nair SK, Burley SK (2003) X-ray structures of MycMax and Mad-Max recognizing DNA. Molecular bases of regulation by proto-oncogenic transcription factors. Cell 112: 193-205. » CrossRef » Pubmed » Google Scholar

21.Nwaka S, Hudson A (2006) Innovative lead discovery strategies for tropical diseases. Nat Rev Drug Discov 5: 941-955. » CrossRef » Pubmed » Google Scholar

22. Párraga A, Bellsolell L, Ferre-D Amare AR, Burley SK (1998) Co-crystal structure of sterol regulatory element binding protein 1a at 2.3 A Resolution Structure 6: 661-672. " CrossRef » Pubmed » Google Scholar

23.Passos S, Carvalho LP, Orge G, Jerônimo SM, Bezerra G, et al. (2005) Recombinant leishmania antigens for serodiagnosis of visceral leishmaniasis. Clin Diagn Lab Immunol 12: 1164-1167.» CrossRef » Pubmed » Google Scholar

24.Perez Victoria FJ, Castanys S, Gamarro F (2003)Leishmania donovani resistance to miltefosine involves a defective inward translocation of the drug. Antimicrob Agents Chemother 47: 2397-2403. " CrossRef » Pubmed » Google Scholar
25.Rosypal AC, Troy GC, Zajac AM, Duncan RB Jr Waki K, et al. (2003) Emergence of zoonotic canine leishmaniasis in the United States: isolation and immunohistochemical detection of Leishmania infantum from foxhounds from Virginia. J Eukaryot Microbiol 50: 691-693.» CrossRef " Pubmed " Google Scholar

26.Rosenthal E, Marty P, del Giudice P, Pradier C, Ceppi C et al. (2000) RetroHIV and Leishmania coinfection: a review of 91 cases with focus on atypical location of Leishmania. Clin Infect Dis 31: 1093-1095. » CrossRef " Pubmed

27.Sundar S, Gupta LB, Makharia MK, Singh MK, Voss A, et al. (1999) Oral treatment of visceral leishmaniasis with miltefosine. Ann Trop Med Parasitol 93: 589-597. » CrossRef » Pubmed " Google Scholar

28.Sundar S, More DK, Singh MK, Singh VP, Sharma S, et al. (2000) Failure of pentavalent antimony in visceral leishmaniasis in India: report from the center of the Indian epidemic. Clin Infect Dis 31: 1104-1108.» CrossRef " Pubmed » Google Scholar

29.Thomas MC, Garcia PJL (2000) Molecular Characterization of KMP11 from Trypanosoma cruzi: A Cytoskeleton-Associated Protein Regulated at the Translational Level. DNA and Cell Biol 19: 47-57. » CrossRef » Pubmed

30.Marsden RL, McGuffin LJ, Jones DT (2002) Rapid protein domain assignment from amino acid sequence using predicted secondary structure. Protein Sci 11: 2814-2824.» CrossRef » Pubmed » Google Scholar

31.Tolson DL, Jardim A, Schnur LF, Stebeck C, Tuckey C, et al. (1994) The kinetoplastid membrane protein 11 of Leishmania donovani and African trypanosomes is a potent stimulator of T-lymphocyte proliferation. Infect Immun 62: 4893-4899. " CrossRef » Pubmed » Google Scholar

32. Venkatachalam CM, Jiang X, Oldfield T, Waldman M (2003) LigandFit: a novel method for the shape-directed rapid docking of ligands to protein active sites. J Mol Graph Model 21:289-307. " CrossRef » Pubmed » Google Scholar

33. Zhang Y, Talalay P, Cho CG, Posner GH (1992) A major inducer of anticarcinogenic protective enzymes frombroccoli: iso-lation and elucidation of structure. Proc Natl Acad Science 89: 2399-403. " CrossRef » Pubmed » Google Scholar 